\title{
Abnormal Colonization of Neonates in an ICU: Conversion to Normal Colonization by Pharyngeal Implantation of Alpha Hemolytic Streptococcus Strain 215
}

\author{
KATHERINE SPRUNT, M.D., ${ }^{(25)}$ GRACE LEIDY, M.A., AND WINIFRED REDMAN, B.A. \\ Division of Infectious Diseases, The Babies Hospital, The Children's Medical and Surgical Center of New York, and \\ the Department of Pediatrics, College of Physicians and Surgeons, Columbia University, New York, New York, USA
}

\section{Summary}

Normal bacterial flora provide a natural defense mechanism against infection. Our experience indicates that "normal" flora for the ICU neonate contains alpha bemolytic $(\alpha-)$ streptococci as the predominant organism. The purpose of the current investigation is to study the feasibility of implanting a carefully selected, naturally occurring strain of $\alpha$-streptococcus in the nasopharynx of neonates considered to be at high risk of infection because of the abnormal colonization of their pharynx with potential pathogens.

Twenty-two infants in the neonatal intensive care unit have received nasopharyngeal implantation with strain 215 a-streptococcus. In 16 infants, $\alpha$-streptococci, including the implant strain in pure or mixed $a$-streptococcal populations, constituted the predominant pharyngeal flora within 48 to $72 \mathrm{hr}$ of implantation. The implant strain was not recovered from the remaining six infants. The procedure was apparently innocuous in this small number of patients.

We conclude that implantation of a streptococcus can be established in the pharynx of infants abnormally colonized with pathogenic organisms in high titer and that successful implantation of strain $\mathbf{2 1 5}$ can result in prompt development of normal pharyngeal flora, with a-streptococci predominant, within $48 \mathrm{hr}$. There has been no evidence of infection or other adverse reaction caused by the implant strain.

Normal bacterial flora provide a natural defense mechanism against infection $(3,4,6)$. Interrelationships among bacteria normally colonizing such sites as the pharynx and gut apparently maintain the normal balance of bacterial populations at the sites $(8,11,16)$. Disruption of these interrelationships by, for example, antibiotic therapy may lead to abnormal colonization of the pharynx with potential pathogens in high titers and subsequent superinfection by them $(10,18)$. Strains of the viridans group of streptococci play an important role in maintenance of the normal balance. They inhibit growth of abnormal colonizers of the pharynx in vitro and apparently in vivo $(1,8,11,12)$ in that their suppression by antibiotics correlates with the occurrence of abnormal colonization $(14,16)$. Deliberate preselection of penicillinresistant alpha hemolytic $(\alpha-)$ streptococci in the pharynx by prior PO penicillin therapy permits persistence of these streptococci during a subsequent period of high-dose penicillin therapy. Their persistence prevents abnormal colonization (14).

In a study of the oropharyngeal bacterial flora of 223 neonates in the Neonatal Intensive Care Unit (ICU), we found that clinical infection occurred in infants with abnormal colonization of the pharynx in high titer and was caused by an organism with the cultural characteristics of the abnormal colonizer. Infants whose throat cultures showed "normal" flora ( $\alpha$-streptococci as predom- inant organism) experienced no infection with the exception of two infants colonized and infected by Staphylococcus aureus (15).

Such information about normal bacterial interrelationships has led to the deliberate implantation of bacteria with desirable characteristics for the protection of animals $(7,19)$ and more recently of man including infants $(5,9,13,17)$. The purpose of the current investigation was to evaluate the implantation of a carefully selected, naturally occurring strain of $\alpha$-streptococcus in the nasopharynx of neonates considered to be at high risk of infection because of the abnormal colonization of their pharynx with potential pathogens in high titer. Twenty-two such infants were given the selected strain.

\section{MATERIALS AND METHODS}

\section{DEFINITIONS}

Normal and "abnormal" bacterial colonization, as defined previously (15) and used in this paper, are broad and arbitrary terms based on total colony-forming units (cfu) and the proportions of types of bacteria comprising the population in cultures of the oropharynx.

In brief, normal colonization indicates alpha hemolytic streptococci as predominant organisms in quantities exceeding $10^{4} \mathrm{cfu} /$ $\mathrm{ml}$ of a one-ml culture sample. Abnormal colonization or bacterial "overgrowth" indicates that the predominant organism (other than $\alpha$-streptococci) is present in concentrations of $10^{4}$ or greater $\mathrm{cfu} / \mathrm{ml}$ and is generally $>90 \%$ of a sample population. This latter definition was modified for $S$. aureus $\left(10^{3} \mathrm{cfu} / \mathrm{ml}\right.$ or more and not necessarily the predominant organism, but $>2 \%$ of the total population) (15).

\section{SELECTION OF ALPHA HEMOLYTIC $(\alpha-)$ STREPTOCOCCI FOR IMPLANTATION}

Criteria adopted for selection of strains for implantation were that they be: (1) obtained from the oropharynx of normal newborn infants who remained healthy during follow-up for at least three months after birth; (2) resistant to 0.5 to 1.0 but not $3.0 \mu \mathrm{g}$ of ampicillin per $\mathrm{ml}$ and sensitive to a number of other commonly used antibiotics; (3) able to inhibit in vitro growth of organisms causing abnormal colonization such as $S$. aureus, enteric type gram-negative bacilli, and Pseudomonas aeruginosa; and (4) consistent with assignment to the mitis group of viridans streptococci.

To select $\alpha$-streptococci meeting these criteria, we examined oropharyngeal cultures from 184 infants at least three days old in the newborn nurseries at the Sloane Hospital for Women, Columbia Presbyterian Medical Center, New York City. Single colonies of $\alpha$-streptococci resistant to at least $0.5 \mu \mathrm{g} / \mathrm{ml}$ of ampicillin were isolated from $26(14 \%)$ of the infants. Of 14 such strains that were 
examined for their in vitro inhibitory capacity by a selective agar overlay method (20), five yielded complete inhibition of the test cultures used ( $S$. aureus, Proteus mirabilis, Ps. aeruginosa, and Klebsiella pneumoniae). Presumably, hydrogen peroxide production was essential for the inhibition because addition of catalase to the media eliminated the inhibitory effect.

Three strains selected for further study showed the following characteristics: autoagglutination with growth in broth, bile insolubility, resistance to optochin and bacitracin (disk test), growth in $2 \%$ but not in $4 \% \mathrm{NaCl}$ in broth, failure to produce ammonia from arginine, and to gel in 5\% sucrose broth; failure to grow in $0.1 \%$

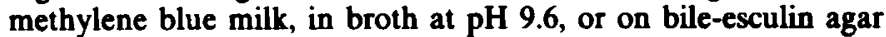
(Difco Laboratories). They produced an acid reaction with sucrose, glucose, lactose, maltose, and mannose but not with raffinose, trehalose, salicin, inulin, and sorbitol. They yielded a precipitate with group $\mathbf{H}$ streptococcal antiserum from Difco Laboratories but not with that from Burroughs Wellcome \& Co. There was no reaction other than that with group $\mathbf{H}$ using the Difco Streptococcus Antisera Set (21) and none with antisera of groups $A, B$ and C (22). No capsular swelling was demonstrated with the Denmark pneumococcal omniserum.

One of these three strains, No. 215 , isolated from an infant known to be healthy for the first three months of life, was arbitrarily selected for implanting. It was susceptible to erythromycin, lincomycin, chloramphenicol, and gentamicin, resistant to kanamycin and oxacillin, and intermediate in susceptibility to ampicillin, penicillin, and cephalothin by the Bauer et al. disk method. It grew on agar containing $1 \mu \mathrm{g}$ and not on agar containing $3 \mu \mathrm{g}$ of ampicillin per $\mathrm{ml}$. It was avirulent for mice when given IP in broth at concentrations up to $1.5 \times 10^{8} \mathrm{cfu}$ (the undiluted broth culture). No capsular swelling was found with rabbit antiserum produced against strain 215 although the strain is antigenic as demonstrated by precipitation tests with the same serum. Strain 215 was preserved by drying in vacuo (2) shortly after isolation.

\section{IMPLANT CULTURE PREPARATION}

Trypticase soy (TS) broth (22) was used for growth. Autoagglutination of the streptococci within 18 to $20 \mathrm{hr}$ of incubation at $35^{\circ} \mathrm{C}$ in room air or with $5 \% \mathrm{CO}_{2}$ in air precluded uniform cfu estimates after thorough mixing (Vortex Jr. Mixer). Incubation under anaerobic conditions [Gas Pak (22)] yielded heavier and more dispersed suspensions after mixing and more predictable estimates of $\mathrm{cfu} / \mathrm{ml}$. Anaerobic growth was adopted after the first use of the implantation procedure. For implantation, an 18- to 20hr TS broth subculture $(2.5 \mathrm{ml})$ was centrifuged for $15 \mathrm{~min}$ at 1500 rpm (IEC model SBV), and the sediment was resuspended to volume in Ringer's lactate solution (23) containing $10 \%$ hyperalimentation fluid (implantation solution). The hyperalimentation fluid was prepared in the Presbyterian Hospital Pharmacy. After thorough mixing, the suspension was diluted 20 -fold in the implantation solution; this dilution yielded a preparation containing approximately $10^{6} \mathrm{cfu} / 0.1 \mathrm{ml}$. Actual doses delivered contained approximately $2 \times 10^{5}$ to $5 \times 10^{6} \mathrm{cfu}$, or from $9 \times 10^{4}$ to $2 \times 10^{6}$ cfu/kilo infant. All but five infants received $>5.5 \times 10^{5} \mathrm{cfu} / \mathrm{kilo}$. One exception, the first infant implanted, received no more than $10^{4} \mathrm{cfu}$.

\section{SELECTION OF INFANTS FOR IMPLANTATION}

At the time of selection for implantation, all infants were abnormally colonized, and no $\alpha$-streptococci were found in their pharyngeal cultures. Early in the series, each implanted infant was facing some additional hazard thought to increase his risk of infection, such as prolonged antibiotic therapy, surgery, intubation, or radiation. Later in the series, infants were included who were abnormally colonized by potential pathogens considered difficult to treat if they caused infection.

Twenty-two infants were selected. All had received antibiotics at some time prior to implantation, one beginning on his 12th day and the remainder within their first or second day of life. Ten were given at least two courses of antibiotics prior to implantation.
Initial diagnoses included respiratory distress syndrome, severe chronic lung disease, group B streptococcal sepsis and meningitis, and neuroblastoma. Six were transfers from another hospital, and one infant was born at home. Nineteen of 22 were males.

\section{IMPLANTATION PROCEDURE}

The principles and process of implantation were discussed with the parents of each infant selected for the procedure. A fully informative consent form was read and signed. Each infant received 0.1 to $0.15 \mathrm{ml}$ of the bacterial suspension placed in the nasopharynx through a No. 5 Fr nasogastric feeding tube. For the procedure, the infant was supported by a hand under the shoulders so that its head fell back. The selected amount of the suspension was drawn into the tubing by suction from a $1.0-\mathrm{ml}$ calibrated syringe, and the tube was inserted along the floor of the nose until it reached the nasopharynx. The suspension was ejected into the nasopharynx, and the tube was "rinsed" with air and withdrawn. The infant was then held with its head back for one-half a minute. Infants did not appear to be disturbed by the process. One of us (Dr. Sprunt) carried out each implantation procedure.

\section{OROPHARYNGEAL CULTURE SAMPLING}

All cultures were obtained by Dr. Sprunt while the infants were in the hospital. A dry nasopharyngeal Calgiswab was wiped across each infant's posterior pharynx and placed in a tube containing 1 $\mathrm{ml}$ of TS broth. The tube was delivered to the laboratory within 10 to 20 min where it was agitated in a vortex shaker, and its swab was discarded. According to the numbers and variety of organisms seen on a gram-stained smear, dilutions were prepared and plated with calibrated loops $(0.01$ and $0.001 \mathrm{ml})$ on trypticase soy (22) agar with $5 \%$ defibrinated horse blood (including plates containing 0.2 and $0.5,1.0$ and $3.0 \mu \mathrm{g}$ of ampicillin per $\mathrm{ml}$ ), mitis salivarius agar with tellurite and MacConkey, mannitol salt, and bile esculin agars (Difco Laboratories). The highest inoculum plated was 0.05 $\mathrm{ml}$ of the undiluted sample. The latter was included if no organisms were seen on a gram-stained smear or for selective agars as indicated. Two or three concentrations were seeded on a single plate, and the plates were incubated for 20 to $24 \mathrm{hr}$ at $35^{\circ} \mathrm{C}$ in $5 \%$ $\mathrm{CO}_{2}$ in air (mannitol salt plates in air). A duplicate set of the blood agar series was incubated anaerobically if an infant's prior culture yielded many colonies of a pseudomonad. Routinely, a ten-fold dilution of a culture sample in TS broth containing 10 to $15 \%$ glycerol (vv) was frozen at $-40^{\circ} \mathrm{C}$ for future reference, if necessary.

Types of colonies were identified by standard methods, and results were expressed as their approximate numbers per $\mathrm{ml}$ of the undiluted culture sample. The term "approximate" is generally omitted in "Results" to limit its repetitive use. Numbers are considered approximate because duplicate plates of a single agar medium were not used, and precise counts of particular types of colonies were at times difficult to determine.

\section{SELECTION OF $\alpha$-HEMOLYTIC STREPTOCOCCI AFTER IMPLANTATION}

Ten to 15 single colony isolates of $\alpha$-streptococci were selected from each pharyngeal culture sample taken after implantation. Colonies morphologically distinct from strain 215 on blood agar or mitis salivarius agar were selected in duplicate. The isolates were seeded into $2.5 \mathrm{ml}$ of blood (1\%) broth incubated overnight, and generally after a second single-colony isolation, the organisms were frozen and later examined for marker characteristics of strain 215. The following marker characteristics were used routinely to indicate identity with strain 215: precipitate formed with group $\mathrm{H}$ (Difco Laboratories) antiserum, resistant to 1 but not $3 \mu \mathrm{g}$ of ampicillin per $\mathrm{ml}$, acid production with maltose, lactose, and mannose, but not with salicin, trehalose, or raffinose and failure to hydrolyze arginine. Isolates differing from the implant strain in at least two of the above characteristics were considered to be not identical. In addition, for each infant implanted, one isolate 
resembling strain 215 was dried in vacuo and tested for its antibiogram, its ability to inhibit growth of test strains, and often, of the infant's own "overgrowth" organism.

\section{RESULTS}

The twenty-two infants in the ICU who received nasopharyngeal implantation with strain $215 \alpha$-streptococcus are grouped for review as follows: group 1 ( 7 infants), those with no $\alpha$-streptococci found in pharyngeal cultures prior to or on the day of implantation and from whom only the implant strain was recovered in the first cultures following implantation; Group 2 (5 infants), those with no $\alpha$-streptococci found in pharyngeal cultures prior to implantation but from whom other $\alpha$-streptococci in addition to the implant strain were recovered after implantation; Group 3 (4 infants), those from whom no $\alpha$-streptococci were recovered except in the culture taken immediately before implanting strain 215 and from whom the implant strain was recovered in mixed $\alpha$-streptococcal flora after implantation; Group 4 (6 infants), the failures, from whom the implant type was not recovered after implantation.

\section{GROUP 1 INFANTS}

Table 1 lists seven group 1 infants, in order of age at the time of implantation, from whom only the implant strain of $\alpha$-streptococcus was recovered in the first days after the procedure was carried out. Dominance of $\alpha$-streptococci within one to three days after implantation developed in all the infants.

Infant 1 was the first of this group implanted. A strain of $\alpha$ - streptococcus other than the implant strain, first isolated on the sixth day, was the dominant $\alpha$-streptococcus on the seventh day. It differed from the implant type in at least four markers.

Implantation was carried out for infant 2 (and infants 4, 6, 14, 15 , and 19) because of high titer colonization by a strain of Staphylococcus epidermidis resistant to multiple antibiotics. The staphylococcus, which was prevalent in the nursery for several months, had caused meningitis in one infant that was exceedingly difficult to treat.

Infant 4 had abnormal colonization with $S$. aureus and $S$. epidermidis. Following antibiotic therapy and prior to implantation, colonization shifted to $S$. epidermidis alone. As noted in the footnote to Table 1, the implant strain was no longer found on the 56th day after implantation. On that day, he received ampicillin and gentamicin for $24 \mathrm{hr}$, following which on the 64th day streptococci with the characteristics of strain 215 predominated. On the 70th day, during another course of ampicillin and gentamicin (66th to 72nd days) and for 19 days thereafter (4 culture samples), all 60 colonies of $\alpha$-streptococci tested had the characteristics of the implant strain.

The $S$. aureus of infant 6 was first recovered the day before and was predominant on the day of implantation.

Infant 7 had received three courses of antibiotics prior to implantation of strain 215 . His slow lactose fermenting Escherichia coli had increased in population density $\left(10^{5}\right.$ to $10^{6} \mathrm{cfu} / \mathrm{ml}$ of culture samples) after each course. A nonhemolytic streptococcus was present in the culture taken on the day he received the implant strain. It represented about $25 \%$ of the sample population and was not found from the sixth through the 20th day after implantation.

Table 1. Patients from whom $\alpha$-streptococci were not recovered prior to implanting strain 215 (only implant type was found for days indicated $)^{1}$

\begin{tabular}{|c|c|c|c|c|c|c|c|}
\hline \multicolumn{2}{|c|}{ Infant } & \multicolumn{2}{|c|}{ Implant day } & \multicolumn{2}{|c|}{ Colonization before implant } & \multicolumn{2}{|c|}{$\alpha$-Streptococci after implant } \\
\hline 1 & $\mathbf{M}$ & 9 & 1580 & E. coli $(>99)$ & 5 & $\frac{(60 \text { to } 70)-85-65}{(2 \text { to } 6)-7-10}$ & 4 \\
\hline 2 & $\mathbf{M}$ & 14 & 1450 & S. epidermidis ${ }^{2}(100)$ & 5 & $\frac{0-20-80-60-98}{1-2-3-(5 \& 7)-10}$ & 5 \\
\hline 4 & $\mathbf{M}$ & 24 & 700 & S. epidermidis $(100)$ & 2 & $\frac{10-50->95}{1-3-(5 \text { to } 14)}$ & 18 \\
\hline 5 & $\mathbf{M}$ & 26 & 1000 & S. aureus $(80->99)$ & 8 & $\frac{1-80-40-8-50}{1-3-5-6-11}$ & 14 \\
\hline 6 & F & 31 & 970 & $\begin{array}{l}\text { S. epidermidis }(100-80-5) \\
\text { S. aureus }(0-20-95)^{3}\end{array}$ & $\begin{array}{r}15 \\
2\end{array}$ & $\frac{10->95}{1-(2 \text { to } 15)}$ & 9 \\
\hline
\end{tabular}

${ }^{1}$ Persistence of implant strain (\% of $\alpha$-streptococcal colonies) and comments: infant 1 , at 39 days, $0.02 \%$. Not found at 53 days and 14 months.

infant 2 , at 25 days, $20 \%$. At three months, $0.05 \%$.

infant 3, at 42 days, $3 \%$. Not found at 8 months.

infant 4 , predominant for 28 days, declined to $1 \%$ at 47 days. Not detected at 56 days.

infant 5, predominant for 33 days. At two months, $0.2 \%$. Variable percentage of $\alpha$-streptococci for 14 days related to proportion of neisseria and

S.aureus colonies. $\alpha$-Streptococci $90 \%$ or more after 14 days ( 8 culture samplings).

infant 6 , predominant for 37 days and $-10 \%$ of $\alpha$-streptococci at 71 days.

infant 7 , at day $17,18 \%$, and at day $27,0.3 \%$. At two months, $0.1 \%$.

Cultures of other sites:

infant 1, rectal swab when 2 days old, $E$. coli: small bowel, $E$. coli (predominant) and klebsiella.

infant 2, before implant, urine and umbilicus at 3 days and stool at 6 days, $S$. epidermidis with same resistance pattern as pharyngeal organism.

infant 4, stool (day implanted), lactose fermenter $98 \%$; $S$. epidermidis (2\%).

infant 5, stool (day implanted), lactose fermenter 90; enterococci 10\%; few S. epidermidis.

${ }^{2} S$. epidermidis-resistant to penicillin, ampicillin, oxacillin, kanamycin, chloramphenicol, erythromycin. Susceptible to gentamicin and cephalothin.

${ }^{3} 0=$ not found. Percentages approximate. 


\section{GROUP 2 INFANTS}

The data of Table 2 are from five group 2 infants from whom no $\alpha$-streptococci were recovered prior to the implantation, but from whom $\alpha$-streptococci differing from the implant in three or more marker characteristics were recovered within the first two days after implantation. One such strain of $\alpha$-streptococcus other than the implant was recovered within the first two days from infants 8, 9, 10, and 11. Two strains differing from the implant (one predominant) were recovered from infant 12 on the second day.

Infant $\mathbf{1 2}$ is particularly interesting in that after a long period (49 days through 5 months) during which the implant strain was not recovered, he received oxacillin at 7 months and ampicillin a month later for lower respiratory tract infection. Following therapy on each occasion, a strain of $\alpha$-streptococcus with the characteristics of the implant strain emerged as a low proportion ( 0.02 and $2 \%$ ) of the streptococcal populations. It was not recovered at 10 months and $1 \frac{1}{2}$ years.

\section{GROUP 3 INFANTS}

The data of Table 3 are from four group 3 infants whose oropharynx yielded $\alpha$-streptococci on the day of, but before, implantation and from whom the implant strain was subsequently recovered. All four infants had had cultures two days before implantation that yielded no $\alpha$-streptococci. No culture was taken the day before implantation. The $\alpha$-streptococci recovered immediately before implanting strain 215 differed from it in three or more markers.

\section{GROUP 4 INFANTS}

The implant strain was not recovered from six "implanted" infants. Failure of implantation can be explained in three of six infants. One was the first infant implanted, a baby severely ill with respiratory distress syndrome and abnormally colonized with $P$ s. aeruginosa. He required long-term intubation and surgery on two occasions. He was given $10^{2}$ cfu of the $\alpha$-streptococcus on two occasions and $10^{4}$ on a third with no perceptible effect on his flora. He died before receiving what we now consider to be an adequate dose of strain 215. A second infant from whom the implant strain was not recovered was treated within $24 \mathrm{hr}$ with chloramphenicol for meningitis caused by his abnormal colonizing $E$. coli. The implant strain is susceptible to chloramphenicol. A third infant was removed from the hospital shortly after implantation was carried out. He was carefully followed by his private physician, who reported that pharyngeal cultures were normal by $48 \mathrm{hr}$ after implantation. We were unable to recover the implant strain in the culture he sent us. We consider implantation of this infant to have failed because of inadequate follow-up and lack of documentation.

The reason for failure to isolate the implant strain from the other three infants in group 4 is harder to understand (Table 4). Infant 17 was abnormally colonized with $K$. pneumoniae following therapy for group B streptococcus sepsis neonatorum. The nonhemolytic streptococcus, present on the day of implantation, predominated and was the only streptococcus found for the next four days. The $K$. pneumoniae declined to less than $1 \%$ of the population samples by 3 days. An in vitro selective agar overlay method showed some inhibition of the $K$. pneumoniae by the nonhemolytic streptococcus. At ten days (outpatient visit), $\alpha$-streptococci were the major component (97\%) of the pharyngeal flora. No colonies resembling the implant strain were found.

The data for infant 18 (Table 4) show that $\alpha$-streptococci appeared two days after the implant, but six days elapsed before they became the major component of the bacterial flora. On the 41 st day, progeny of one colony selected resembled strain 215 in its routine marker characteristics.

Infant 19 had group B streptococcal sepsis and meningitis. After ampicillin and gentamicin therapy for $\mathbf{4}$ days and while he was on penicillin therapy, group B streptococci predominated in his oropharynx along with $E$. coli and $S$. epidermidis. On the day of implantation, $\alpha$-streptococci had appeared spontaneously. The $\alpha$ streptococci were resistant to $5 \mu \mathrm{g}$ of ampicillin per $\mathrm{ml}$ and differed from the implant in three other markers. This strain, present

Table 2. Patients from whom no $\alpha$-streptococci were recovered prior to implanting strain 215 . Percent of $\alpha$-streptococci of implant type varied after implantation ${ }^{1}$

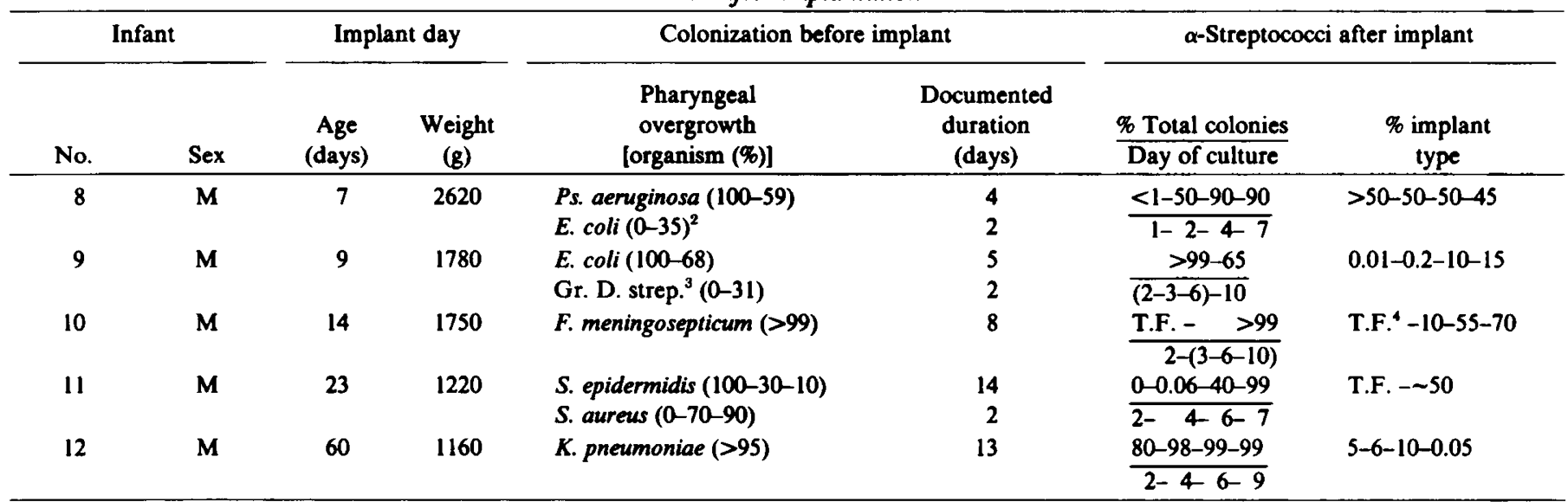

\footnotetext{
' Persistence of implant strain (\% of $\alpha$-streptococcal colonies) and comments: infant 8 , no culture after 7 days.

infant 9,13 days, $(0.8 \%)$. No further cultures.

infant 10 , two months $(0.2 \%)$. Not found at 7 months.

infant 11 , about $3 \%$ at 49 days. No further cultures.

infant 12 , not found 11 to 19 days. 31 days $<0.1 \%$. Not recovered 49 and 84 days and 5 months.

Cultures at other sites:

infant 8 , stool culture 2 days prior to implant showed many lactose fermenters and non-lactose fermenters. infant 10 stool before implant, $F$. meningosepticum predominant.

infant 11 , stool culture before implant, moderate no. $S$. epidermidis (resistant to ampicillin and kanamycin). E. coli predominant.

${ }^{2} 0=$ Not found

${ }^{3}$ not enterococci

\T.F. Too few colonies to assess percentage. Implant and at least one other strain present.
} 
Table 3. Patients from whom $\alpha$-streptococci were recovered on day of implantation, implant type recovered ${ }^{1}$

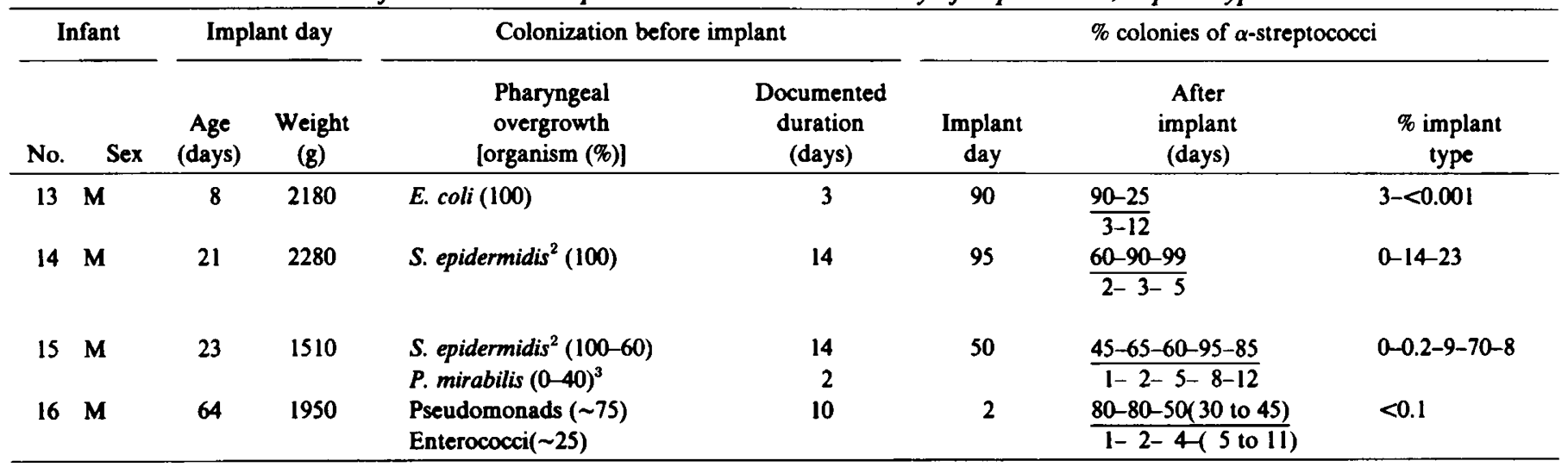

'Infant 13, rectal swab before implant, $E$. coli predominant ( $>99 \%)$; infant 14, implant type recovered at 30 (3\%) and 51 (4\%) days. No further cultures. Stool culture before implanting, $S$. epidermidis predominant ( $>99 \%$ ); infant 15 , implant type about $8 \%$ days 15 and 22 ; not found at one month. Stool culture day of implant, Proteus predominant, $S$. epidermidis present; infant 16, implant type not found at 18, 30, 38, and 47 days. Haemophilus parahaemolyticus and/or Neisseria sp. emerged after implantation.

${ }^{2} S$. epidermidis. Antibiogram similar to that of infants 2, 5, and 6 (Table 1).

${ }^{3} 0$, not found.

Table 4. Patients from whom implant strain was not recovered

\begin{tabular}{|c|c|c|c|c|c|c|c|}
\hline \multirow[t]{2}{*}{102} & \multicolumn{2}{|c|}{ Infant } & \multicolumn{2}{|c|}{ Implant day } & \multicolumn{2}{|c|}{ Colonization before implant } & \multirow[t]{2}{*}{$\begin{array}{l}\alpha \text {-Streptococci } \\
\text { after implant }\end{array}$} \\
\hline & No. & Sex & $\begin{array}{c}\text { Age } \\
\text { (days) }\end{array}$ & $\begin{array}{c}\text { Weight } \\
\text { (g) }\end{array}$ & $\begin{array}{c}\text { Pharyngeal } \\
\text { overgrowth } \\
\text { [organism (\%)] }\end{array}$ & Implant day & \\
\hline 17 & $\mathbf{F}$ & 12 & 2850 & & & $\begin{array}{l}60 \\
40\end{array}$ & $\frac{0-97}{(1-4)-10}$ \\
\hline 18 & $\mathbf{M}$ & 20 & 2500 & & $\begin{array}{l}(0-99) \\
-1)\end{array}$ & $\begin{array}{l}35 \\
65\end{array}$ & $\frac{7-6-29-15-67}{2-3-4-5-6}$ \\
\hline 19 & $\mathbf{M}$ & 21 & 2000 & $\begin{array}{l}\text { C } \\
S \\
E \\
\text { n } \\
\text { o }\end{array}$ & $\begin{array}{l}\text { occi }(0-98-50) \\
00-2-3) \\
-1)\end{array}$ & $\begin{array}{r}5 \\
1 \\
1 \\
55 \\
36\end{array}$ & $\frac{18-7-14-5}{1-4-6-7}$ \\
\hline
\end{tabular}

\footnotetext{
${ }^{1}$ Infant 17, Nonhemolytic streptococcus predominant ( 85 to 97\%) days 1 to 4 after implant. Stool 2 days before, lactose fermenter resembling klebsiella. On $1 \mathrm{M}$ penicillin before and for 4 days after implant; infant 18, Ps. aeruginosa and $K$. pneumoniae predominant for 5 days postimplant. $\alpha$ streptococci resistant to ampicillin $(0.2$ to $0.5 \mu \mathrm{g} / \mathrm{ml})$. $\alpha$-streptococci predominant days 6 through 41 ; infant 19 , $\alpha$-streptococci resistant to $5 \mu \mathrm{g}$ of ampicillin per $\mathrm{ml}$ were present at implantation and differed from the implant in three other marker characteristics. $S$. epidermidis predominant day 4 to 11 after implanting. iv penicillin started, day 12. Rectal swab day before implant, Group D streptococci, lactose fermenters, few $S$. epidermidis.

${ }^{2} 0=$ not found.

${ }^{3} S$. epidermidis resistant to ampicillin, chloramphenicol, kanamycin, erythromycin, and oxacillin.
}

immediately before and after the implant, was a limiting factor in attempts at recovery of the implant strain. Although this original strain declined to $<10 \%$ of the $\alpha$-streptococcal cfu by six days and $1 \%$ or less by 18 days, no streptococci resembling the implant were isolated.

\section{INCIDENCE OF STRAINS RESEMBLING 215 IN THE ICU POPULATION}

Identification of the "implant strain" in infant pharyngeal flora raises the question of how frequently $\alpha$-streptococci with characteristics of strain 215 may be recovered in the ICU. Over a 10month period, culture samples from $180 \mathrm{ICU}$ infants were screened not only for abnormal colonization but also for ampicillin-resistant $\alpha$-streptococci by plating a 1000 -fold dilution ( $10^{-3}$ loop) of the undiluted one-ml culture sample on media that included horse blood agar with and without $0.5 \mu \mathrm{g}$ of ampicillin per $\mathrm{ml}$. Five (2.8\%) of 180 infants yielded streptococci with characteristics of the implant strain. These five were among the 167 infants (93\%) whose cultures yielded $\alpha$-streptococcal populations in the range $10^{5}$ to $10^{6} \mathrm{cfu} / \mathrm{ml}$, a range adequate for assessing numbers of ampicillin-resistant streptococci within the first three days of implantation of all the infants except infant 16 (Table 3).
Of 39 infants from whom $\alpha$-streptococci resistant to at least 0.5 $\mu \mathrm{g}$ of ampicillin per $\mathrm{ml}$ were recovered, 28 yielded streptococci resistant to one $\mu \mathrm{g} / \mathrm{ml}$. Five of the latter group or $3 \%$ of the total of the above 167 infants resembled the implant strain in the routine marker characteristics used in assessing colonies of $\alpha$ streptococci from the implanted infants. Isolation of the implant strain from 16 of 22 infants implanted or 16 of 19 , omitting the three failures described earlier, is noteworthy.

\section{DISCUSSION}

Implantation was clearly responsible for conversion of the flora to normal in the seven infants listed in Table 1 in that the implant strain was the only strain of $\alpha$-streptococcus recovered from 4 to 18 days after implantation. The role of the procedure for the next five infants is less clear cut (Table 2). Although no streptococci were found in the culture before implantation and cultures were normal within $48 \mathrm{hr}$ after the procedure, strain 215 made up only part of the postimplant streptococcal population. The rapid appearance and preponderance of other streptococcal strains, apparently in conjunction with implantation, may indicate that the process of implantation augments or initiates natural selective 
mechanisms of the pharyngeal mucosa for colonization by streptococci. Rapid conversion of the pharyngeal flora to normal, however, also occurs spontaneously, as demonstrated by three of 22 infants (infants 13, 14 and 15, Table 3) who developed a preponderance of $\alpha$-streptococci within the $48 \mathrm{hr}$ prior to the implant procedure.

Similar rapid and spontaneous streptococcal colonization has been observed in other infants abnormally colonized by gramnegative bacilli who were not implanted. This pattern of flora development is not rare but would be unlikely to occur on a definite schedule unless influenced by the implantation process. A slower rate of change in the pattern of colonization is also observed. Infant 18 (Table 4) probably represents this pattern of normal colonization in that $\alpha$-streptococci (not strain 215) did not become predominant until the sixth postimplant day. Streptococci vary considerably in their inhibitory capacity for overgrowing strains as tested in vitro $(8,11,20)$. It seems reasonable that the first strains acquired by an infant influence the rate and consistency of development of a normal flora pattern.

The reasons for failure of implantation in infants 17,18 , and 19 (Table 4) are not clear. It may be significant that these unexplained failures all occurred in infants who received relatively small amounts of the implant strain. The three failures were among the five infants given less than $5.5 \times 10^{5} \mathrm{cfu} / \mathrm{kilo}$ of body weight. The dosage used may have been inadequate.

The implant strain was selected for its ability to inhibit in vitro potential pathogens causing abnormal colonization in the nursery and for its degree of resistance to ampicillin. The latter criterion was considered potentially useful in that $\alpha$-streptococci resistant to 1 unit and sensitive to 3 units of penicillin were found and persisted in the pharynx of adults given high doses of peniciliin IV; streptococcal persistence apparently prevented abnormal colonization (14). It should be possible, therefore, for the implant strain to persist through subsequent courses of antibiotics or to be selected by future therapy and maintain a normal pattern of pharyngeal flora for the implanted infant. Two opportunities for observation of possible survival and selection have occurred. Subsequent therapy at 8 and 9 months may have selected the $\alpha$ streptococci with the characteristics of strain 215 on two occasions in infant 12 (Table 2) from whom no similar colonies had been recovered from 49 days through 5 months after implantation. After such a long period of time, appearance of the strain could represent a late coincidental acquisition of a similar streptococcus, but a similar coincidence occurring on two occasions is most unlikely. Selection of the strain by antibiotics is clearer, however, for infant 4 (Table 1). The implant strain had slowly decreased in proportion to the total streptococcal population until it was no longer found on the 56th day. In the next culture, eight days after a 24-hr exposure to ampicillin and gentamicin, streptococci with the characteristics of strain 215 predominated, and during another course of ampicillin and gentamicin (66th to 72nd days), only the implant type was recovered. The course of this patient suggests strongly that the organism can persist undetected in the pharynx and can be selectively augmented by antibiotic therapy.

Several conclusions can be drawn from the experience presented. Implantation of a streptococcus can be achieved in the infant pharynx in the presence of abnormal colonization in high titer. Successful implantation of strain 215 can result in prompt development of normal pharyngeal flora, i.e., $\alpha$-streptococci predominant within $48 \mathrm{hr}$. There has been no evidence of disease or other adverse reaction caused by the implant strain. Admittedly, the number of patients (22) exposed to it is small (24).
We cannot determine as yet whether artificially acquired normal flora provides as effective a host defense mechanism against infection as naturally acquired normal flora apparently does. A controlled study is under way to answer this question.

\section{REFERENCES AND NOTES}

1. Aly, R., Maibach, H. I., Shinefield, H. R., and Mandel, A. D.: Protection of chicken embryos by viridans streptococci against the lethal effect of Staphylo. coccus aureus. Infect. Immun., 9: 559 (1974).

2. Brown, J. H.: Vacuum tubes for storage and shipment of bacteria. Science, 64: 429 (1926).

3. Dineen, P.: The effect of reduction of bowel fora in experimental staphylococcal infection in mice. Proc. Soc. Exp. Biol. Med., 104: 760 (1960).

4. Dubos, R. J., and Schaedler, R. W.: The effect of the intestinal flora on the growth rate of mice and on their susceptibility to experimental infections. $J$. Exp. Med., 111: 407 (1960).

5. Ehrenkrantz, N. J.: Bacterial colonization of newborn infants and subsequent acquisition of hospital bacteria. J. Pediatr., 76: 839 (1970).

6. Freter, $R$.: The fatal enteric cholera infection in the guinea pig achieved by inhibition of normal enteric flora. J. Infect. Dis., 97: 57 (1955).

7. Freter, R., and Abrams, G. D.: Function of various intestinal bacteria in converting germ free mice to the normal state. Infect. Immun., 6: 119 (1972).

8. Johanson, W. G., Blackstock, R., Pierce, A. K., and Sanford, J. P.: The role of bacterial antagonism in pneumococcal colonization of the human pharynx. J. Lab. Clin. Med., 75: 946 (1970).

9. Lodinova, R., Jovja, V., and Wagner, V.: Serum immunoglobulins and coproantibody formation in infants after artificial intestinal colonization with Escherichia coli 083 and oral lysozyme administration. Pediatr. Res., 7: 659 (1973).

10. Louria, D. B., and Brayton, R. G.: The efficacy of penicillin regimens with observations on the frequency of superinfection. J. Am. Med. Assoc., 186: 787 (1963).

11. Sanders, E.: Bacterial interference. 1. Its occurrence among the respiratory tract flora and characterization of inhibition of group $A$ streptococci by viridans streptococci. J. Infect. Dis., 120: 698 (1969).

12. Sanders, C. C., Nelson, G. E., and Sanders, W. E., Jr.: Bacterial interference. IV Epidemiological determinants of the antagonistic activity of the normal throat flora against group A streptococci. Infect. Immun., 16: 599 (1977).

13. Shinefield, H. R., Wilsey, J. D., Ribble, J. D., Boris, M. X., Eichenwald, H. F. and Dittman, C.: Interactions of staphylococcal colonization. Influence of normal flora and antimicrobials on inoculated Staphylococcus aureus strain 502A. Am. J. Dis. Child., 111: 11 (1966).

14. Sprunt, K., Leidy, G., and Redman, W.: Prevention of bacterial overgrowth. J. Infect. Dis., 123: 1 (1971).

15. Sprunt, K., Leidy, G., and Redman, W.: Abnormal colonization of neonates in an intensive care unit: means of identifying neonates at risk of infection. Pediatr. Res., 12: 998 (1978).

16. Sprunt, K., and Redman, W.: Evidence suggesting importance of role of interbacterial inhibition in maintaining balance of normal flora. Ann. Intern. Med. 68: 579 (1968).

17. Symposium on Oral Enteric Bacterial Vaccines. Acta Microbiol. Acad. Sci. Hung., 21: 1 (1974).

18. Tillotson, J. R., and Finland, M.: Bacterial colonization and clinical superinfection of the respiratory tract complicating antibiotic treatment of pneumonia. J. Infect. Dis., 119: 597 (1969).

19. Wickman, K.: Studies of bacterial interference in experimentally produced virus in guinea pigs. Acta Pathol. Microbiol. Scand. Sect. Microbiol., 78: 15 (1970).

20. Method in brief. Pour plate preparations of strains of the viridans group of streptococci in the range of $10^{3}$ to $10^{4}$ cfu were incubated for 20 to $24 \mathrm{hr}$ in trypticase soy agar containing $2 \%(\mathrm{vv})$ heat inactivated horse serum $\left(65^{\circ} \mathrm{C}\right.$ for $1 / 2 \mathrm{hr}$ ) and with (and without) a fermentable sugar (e.g., lactose) added to $1 \%$ (vv). After incubation, an equal volume of a selective agar, with (and without) catalase was overlayed (mannitol salt agar for staphylococci; MacConkey agar for gram negative enteric bacilli or pseudomonads). After hardening, approximately $10^{4}$ to $10^{5} \mathrm{cfu}$ of a faintly turbid young culture of a test strain was spread on the surface of the appropriate agar overlay and the plates incubated in air at $35^{\circ} \mathrm{C}$ for 20 to $24 \mathrm{hr}$.

21. Groups A, B, C, D, E, F, G, H, K, L, M, N, O, mg streptococci.

22. Baltimore Biological Laboratories,

23. Lactated Ringer's injection, USP.

24. Thirty-three infants have now received strain 215 without adverse reaction.

25. Requests for reprints should be addressed to: Katherine Sprunt, M.D., Box 50 Babies Hospital, 167th Street and Broadway, New York City, N.Y. 10032 (USA).

26. This research was supported by a SCOR Grant HL 14218, NHLI.

27. Received for publication October 10, 1978.

28. Accepted for publication May 15, 1979. 